\title{
Quantum Computation Based on Retarded and Advanced Propagation
}

\begin{abstract}
Giuseppe Castagnoli*
Computation is currently seen as a forward propagator that evolves (retards) a completely defined initial vector into a corresponding final vector. Initial and final vectors map the (logical) input and output of a reversible Boolean network respectively, whereas forward propagation maps a one-way propagation of logical implication, from input to output.

Conversely, hard - NP-complete - problems are characterized by a twoway propagation of logical implication from input to output and vice versa, given that both are partly defined from the beginning. Logical implication can be propagated forward and backward in a computation by constructing the gate array corresponding to the entire reversible Boolean network and constraining output bits as well as input bits. The possibility of modeling the physical process undergone by such a network by using a retarded and advanced in time propagation scheme is investigated.
\end{abstract}

PACS numbers: 89.70.+c, 02.50.-r, 03.65.-w, 89.80.+h.

\section{THE NOTION OF COMPUTATION REVERSIBILITY}

This work deals with the possibility of deepening the notion of computation reversibility in the quantum framework. This might provide a richer form of computation.

The discovery that computation can be reversible in principle and the development of the thought model of a classical reversible computer, became cornerstones of research on the physical basis of computation $[1,2,3,4]$. Once found to be reversible, computation could be moved into the quantum framework $[5,6,7,8,9]$.

\footnotetext{
*Information Technology Dept., Elsag Bailey, 16154 Genova, Italy
} 
The finding that the deeper the physical basis the richer is the form of computation, has been a remarkable result:

- a quantum register undergoing a unitary evolution can perform in quantum superposition (or parallelism) a number of computations which grows exponentially with register size,

- superposition elements interfere and the successive measurement brings about a collective property of those elements in the episystem,

- this enables faster than classical computation in some special instances $[7,10,11,12$, $13,14]$.

Although essential to bring computation into the quantum domain, the notion of computation reversibility entered the quantum framework unaltered. This, we argue, might constitute an undue restriction.

As is well known, the notion of reversibility, thus of the absence of a privileged direction of causality, can undergo a quantum change. Models that postulate the non-redundant coexistence of a retarded and an advanced propagation in the quantum framework have been formulated $[15,16,17,18,19]$, although mostly outside a computational context.

FIG. 1.

Let us examine the notion of computation under a broader perspective, keeping in mind that it can be mapped on either a one or a two-way propagation. Currently, quantum computation is seen as a propagator that evolves (retards) a completely defined initial vector into a final vector, which is therefore a function of the initial vector. One-way physical propagation maps the one-way propagation of logical implication from the inputs to the outputs of a reversible Boolean network (Fig. 17a). Such a network appears in the space-time diagram of a "sequential computation" process: network nodes are the register qubits before and after each transformation, Boolean gates and wires represent the transformations. 
The existence of a privileged input-output direction (of one-way propagation) in sequential computation sets some constraints on the underlying Boolean network. A Boolean constant must be preassigned to any input node, whereas none is allowed on output nodes. An output cannot be connected to an upstream input which participates in the logical determination of that same output (Fig. [ $\mathrm{b} b$; ignore dotted arrow). The fact that sequential computation is suspected to be unable to solve NP-complete problems in polynomial time [20] might be related to the presence of such constraints.

Conversely, Boolean networks do appear in problem solving in a less constrained way. An example is the problem of finding the satisfiability of a generic Boolean network. Fig. 11c exemplifies the standard way of stating this problem for a reversible network. Out of habit, the two extremities of the network are still called input and output, although there is no longer a privileged input-output direction. Both the input and the output must be partly constrained: constant Boolean values must be assigned to part of the input nodes, another part must be left free. The same applies to the output. The Boolean constants applied to both the input and the output propagate a conditional logical implication, respectively in the input-output and output-input directions (of course the logical implication, say, in the inputoutput direction is conditioned by the possible Boolean assignments of the unconstrained part of the input, and similarly for the other direction). The coexistence of two directions of conditional implication makes the problem of determining network satisfiability hard, namely NP-complete.

The case of the Boolean network in Fig. 11b (ignore the time axis) is similar. The output-input connection introduces another form of coexistence of a forward and backward conditional implication (along a loop), which makes the network satisfiability test equally hard.

It is physically quite possible to propagate a conditional logical implication both forward and backward along a reversible Boolean network, by laying out the entire network spatially and by constraining part of the inputs as well as part of the outputs (Fig. 11d). Thus network nodes $r, s, \ldots$ become coexisting qubits $\left|\chi_{1}\right\rangle_{r},\left|\chi_{2}\right\rangle_{s}, \ldots$, (with $\chi_{i}=0,1$ ) as time is 
now perpendicular to the network layout.

We will develop a thought model of this network by applying a sort of divide-and-rule strategy, meant to take advantage of a quantum symmetry related to particle statistics

Conceptually, gates, with their input and output qubits, are first thought of as being "divided", all the constraints imposed on them through the wires are removed. Each independent (unwired) gate is implemented by a quantum transposition of simulated annealingnamely ground state computation. At equilibrium, each gate sits in a degenerate ground state. This can host any linear combination of those gate eigenstates - each a tensor product of the gate qubits eigenstates - which satisfy the gate Boolean relation. Disregarding wires, all gates would relax toward ground in parallel and independently of each other. This, at equilibrium, would produce a network state consisting of a superposition of tensor products which satisfy the Boolean relations imposed by the gates, while those imposed by the wires are ignored.

Wires are restored by using a special symmetry induced by particle statistics. With no loss of generality, each wire is assumed to comprise a NOT function. As such, it will be called $r s$

link. The truth table of link, say, $r, s$ is thus 01 . Such a relation between the eigenvalues of 10

any two interlinked qubits $r, s$, will be imposed by a special symmetry $A_{r s}$, related to particle statistics [defined by $A_{r s}|\chi\rangle_{r}|1-\chi\rangle_{s}=|\chi\rangle_{r}|1-\chi\rangle_{s}, A_{r s}|\chi\rangle_{r}|\chi\rangle_{s}=0$, with $\chi=0,1$ - see Section II.C]. Such symmetries, or their product for all links $\tilde{A}=\prod_{r, s} A_{r s}$ (the $A_{r s}$ pairwise commute), "rule" by restoring consistent wiring and network completeness.

This kind of wiring is liable of two interpretations, one holding in the conventional oneway propagation model, the other in a two-way propagation model (by "propagation", when

\footnotetext{
${ }^{1}$ So far quantum computation has used special features like superposition and multiparticle interference, certainly not particle statistics. The possibility of exploiting particle statistics is investigated in this work.
} 
not explicitly referred to conditional logical implication, we will mean the physical process). Both will be pursued throughout this work.

In one-way propagation, one obtains a quantum annealing computation over a Boolean network. However, no advantage will be found over classical annealing (disregarding quantum tunnelling, not addressed in this work).

In two-way propagation, it is as if $\tilde{A}$ induced a watchdog effect on the overall network state $|\psi(t)\rangle$ generated by the relaxation process of the independent gates. By the way, we should note that $|\psi(t)\rangle$ is not necessarily a pure state, we will use the method of random phases. Symmetrization under $\tilde{A}$ would operate as projection, canceling so to speak the amplitudes of those network eigenstates which would violate $\tilde{A}$, and reinforcing the others through renormalization. This would yield (roughly) to a population of gates relaxing independently of each other, without ever incurring frustration, with an essential advantage over classical annealingt?

In this scheme, any particle statistics symmetry $S$ (for example, singlet or triplet state symmetry) must be seen as a projector $S$ applying to the evolving state for all times $t$ : $\forall t: S|\psi(t)\rangle=|\psi(t)\rangle$. In this view, $S$ does not need to be an initial condition conserved as a constant of motion. Eventually, $S$ commutes with the propagator of $|\psi(0)\rangle$; but this can be so in a reversed perspective, where the propagator could be forged by projection.

Considering a particle statistics symmetry $S$ as the result of projection, is consistent with the usual interpretation where symmetry is seen as a conserved initial condition. However, the two interpretations are not necessarily equivalent: in the current computation context, the projection view appears to favour a two-way propagation model. We mean those interpretations of quantum mechanics which postulate that propagations are driven by both their

\footnotetext{
${ }^{2}$ To some extent, this is similar to present-day quantum error correction, where the onset of errors and decoherence is countered by repeated projection of a redundant computation state onto some symmetric subspace $[21,22,23,24]$.
} 
initial and final conditions ${ }^{[15,16,17,18,19,33]}$.

In current assumptions we are dealing with a set of independently relaxing gates, whose overall state, for all times $t$, is projected on the Hilbert subspace which satisfies symmetry $\tilde{A}$ : propagation would thus be "driven" by relaxation and "forged" by projection.

Let us consider first the usual one-way propagation model. Here propagations are modeled by calculus, which is of course one-way. Any infinitesimal relaxation should be submitted to projection, and so on repeatedly. However, this yields to a Zeno effect that "freezes" the propagation in its initial state: $\forall t:|\psi(t)\rangle=|\psi(0)\rangle$ (Section II.D).

It might be argued that one-way calculus is not fit to model a propagation "forged" by projection. Intuitively, the nature of projection appears to be discontinuous; even if projection is applied at all times $t$, this does not necessarily mean that it can be used in a differential way. As a matter of fact, the Zeno effect disappears when this projection-forged propagation is modeled by the two-way propagation scheme developed in [18].

Here the propagation of $|\psi(t)\rangle$ turns out to be driven by the final condition that projection at all times $t$ yields a state that simultaneously (under a system of mathematically simultaneous equations) approaches ground, according to the independent gate relaxation process, and satisfies $\tilde{A}$.

Interestingly, projection on a predetermined outcome implies an elusive chronology violation. At any time $t_{1}$ the propagation has to choose between two possible projection outcomes, one satisfying, the other not satisfying $\tilde{A}$. Satisfaction of $\tilde{A}$ is the condition of that choice. The propagation meets this condition immediately after the choice: the choice should be before projection (at time $t_{1}$ ), and the condition is met after projection (at time $t_{2}$, reasonably $\left.t_{2}>t_{1}\right)$. Chronology violation is elusive since it can be confined within a time interval $\Delta t=t_{2}-t_{1}$ as short as desired. It can be, so to speak, "safely" confined within a time interval $\Delta t$ such as $\Delta t \Delta E \cong \hbar$, where the ordering of time and causality becomes fuzzy. However, the consequences of choices taken in $\Delta t$ can naturally be "long range". For the sake of clarity, we should note that chronology violation would become completely evident 
if the vector to be projected on a predetermined outcome were discretely different from it. A condition set in the future would determine a choice in the past. It becomes elusive when projection is performed for all $t$.

Section II provides a thought model based on the above notions. It is not an implementation model; some Hamiltonians are represented by Hermitean matrices. However, it appears to be plausible in principle. If implementable, it could speed up the solution of an NP-complete problem with respect to known classical computation.

An analogy can be observed between this work and D. Deutsch's work on closed time-like lines [26]. Their relation to general relativity is irrelevant in this context - what is relevant is the presence of loops of conditional implication. In Fig.1b, forward and backward conditional implications coexist on the two halves of a loop which (in Deutsch's model) becomes a closed time-like line in a chronology violating region (consider now the dotted arrow). Sequential computation circulates along this line. This enables, without necessarily implying it, faster than classical computation (the problem was finding the fixed points of a hard to invert function).

\section{THOUGHT MODEL}

\section{A. Classical Simulated Annealing}

With a view to introducing quantum annealing, we shall review a simple application of classical simulated annealing - also called ground state computation - to the satisfiability problem. Network nodes (Fig. 1d) can be seen as cups, each containing a coin in either head or tail state (say Boolean 0 or 1). Computation follows an if/then process:

(1) does the coin configuration satisfy all the local relations imposed by gates and links?

(2) If YES, stop: a solution has been found and the network is satisfiable.

(3) If NOT, "shake" (randomly flip coins) and go to (1). 
Network unsatisfiability is ascertained with any desired confidence level by looping for a sufficiently large number of times, without ever finding a solution.

In order to speed up computation, each network element (N.E.) should be viewed as a mechanism whereby all states which satisfy the N.E. belong to a degenerate ground state, while the energy level of the others is discretely above ground (Fig. [1d). This feature during "shaking" - provides an energy gradient, orienting the evolution towards ground state. Dumping is of course needed. A heat bath of suitable temperature can provide for both dumping and shaking.

Shaking is required because this kind of computation is subject to "frustration". A coin configuration can satisfy most N.E. and still be far from any solution. The process, so to speak, should be undone. This boils down to the presence of relative energy minima "shaking" is needed to avoid state trapping in such minima. Let $\mathrm{N}$ be the number of network nodes. The number of relative minima and relaxation time - expected time to reach the absolute minimum - is exponential in N, according to today's knowledge by any classical simulated annealing strategy. For a description of efficient strategies, see [27].

\section{B. Quantum Annealing - Gates}

In quantum annealing, the Hamiltonian of the Boolean gate operating on qubits, say, $s, t, u, v$ (Fig. 11d), has 16 eigenstates, namely all possible tensor products of the gate qubit eigenstates. Those tensor products which satisfy the gate belong to a degenerate ground state, while those which do not satisfy it are metastable states belonging to discretely higher, say by more than $\Delta E$, energy levels. An external input or output with a preassigned Boolean

value becomes a one-qubit gate, and that value will correspond to the ground state of the gate Hamiltonian (Fig. 11d).

Given a heat bath temperature $T$ such that $0<k T<<\Delta E$, in a semiclassical picture the probability $p$ of finding (as the result of a measurement) the gate in ground state by the time $t$ follows asymptotically the law 


$$
p=1-e^{-\sigma t},
$$

where $\sigma>0$. Of course this exponential law holds when relaxation has reached a constant rate. Model Hamiltonians of elementary Boolean gates with coexisting inputs and outputs can be found in [28]. Of course such gates differ from the gates used in the sequential approach, where inputs and outputs are successive states of the same register $[6,7,29,30$, $31,32]$.

Links could be implemented accordingly. Then, link-gate interaction Hamiltonians should be added. The number of local minima and computation time would be likely to remain exponential in $N$ (the benefit deriving from quantum tunnelling through local minima is not considered in this work). Links will be dealt with in a different way.

\section{Quantum Annealing and Particle Statistics - Links}

The correlation between simultaneous qubit eigenvalues imposed by links will rely on a special particle statistics symmetry - seen as a projection operator (Section I). The (thought) physical situation giving origin to such symmetry (one for each link) is still obtained by means of a relaxation process.

Without any restriction, a network topology is adopted where nodes come in interlinked pairs (Fig. 1). The "divide" rule is still applied. By "turning off" gate Hamiltonians, links are, so to speak, "ungated". This leaves us with a set of independent links, which comprise all the nodes of the network.

For the sake of visualization, each link node $r, s$ (Fig. Ild) can be seen as a site of a spatial lattice. These sites are labeled by $\lambda=r, s$. Each site hosts exactly one spin $\frac{1}{2}$ particle, with its $\sigma_{z}$ spin component either down $(\chi=0)$ or up $(\chi=1)$. Therefore qubit $|\chi\rangle_{\lambda}$ reads: the $\sigma_{z}$ component of the particle hosted by site $\lambda$ is $\chi$. However, we should like to keep this model abstract: $\lambda$ and $\chi$ can be any two binary compatible attributes (dynamical variables) of a fermion. 
In a particle label representation, qubit $|\chi\rangle_{\lambda}$ is written $|\chi\rangle_{1}|\lambda\rangle_{1}$; for example $|0\rangle_{r}$ becomes $|0\rangle_{1}|r\rangle_{1}$. By this we mean that the $\chi$ attribute of particle 1 has eigenvalue 0 and that its $\lambda$ attribute has eigenvalue $r$. Or, since we are dealing with two identical particles, particle 1 is interchangeable with particle 2; in this case $|0\rangle_{r}$ becomes $|0\rangle_{2}|r\rangle_{2}$. Therefore, qubit representation will be the outcome of a finer identical particle representation.

The lattice $L$ of network nodes (or qubits), with exactly one fermion per node (one independent $\chi$ attribute per qubit), is created as follows. Gate Hamiltonians are still "turned off". Link Hamiltonians, thus completely independent of each other, are "turned on". Then links are allowed to relax on their ground states.

The Hamiltonian $H_{12}^{\lambda}$ of link $r, s$ is defined as follows. It operates on the spatial coordinates of fermions 1 and $2-$ not on their spin. Therefore $H_{12}^{\lambda}$ operates on the Hilbert space:

$$
H_{\lambda}=\operatorname{span}\left\{|r\rangle_{1}|r\rangle_{2},|s\rangle_{1}|s\rangle_{2}, \frac{1}{\sqrt{2}}\left(|r\rangle_{1}|s\rangle_{2}-|s\rangle_{1}|r\rangle_{2}\right), \frac{1}{\sqrt{2}}\left(|r\rangle_{1}|s\rangle_{2}+|s\rangle_{1}|r\rangle_{2}\right)\right\}
$$

where $|r\rangle_{1}|r\rangle_{2}$ reads particle 1 in site $r$, particle 2 also in site $r$, etc.

The energy eigenvalues $\leftrightarrow$ eigenstates of the Hamiltonian

$$
\begin{aligned}
H_{12}^{\lambda}= & \left(\begin{array}{cccc}
E^{\lambda} & 0 & 0 & 0 \\
0 & E^{\lambda} & 0 & 0 \\
0 & 0 & 0 & 0 \\
0 & 0 & 0 & 0
\end{array}\right) \text {, with } E^{\lambda} \text { discretely above 0, are } \\
& E^{\lambda} \leftrightarrow|r\rangle_{1}|r\rangle_{2} \\
E^{\lambda} & \leftrightarrow|s\rangle_{1}|s\rangle_{2} \\
0 & \leftrightarrow \frac{1}{\sqrt{2}}\left(|r\rangle_{1}|s\rangle_{2}-|s\rangle_{1}|r\rangle_{2}\right) \\
0 & \leftrightarrow \frac{1}{\sqrt{2}}\left(|r\rangle_{1}|s\rangle_{2}+|s\rangle_{1}|r\rangle_{2}\right)
\end{aligned}
$$

When link $r, s$ has relaxed onto the ground state of $H_{12}^{\lambda}$, each link node or qubit is associated with exactly one particle. This creates the "lattice" of qubits $r$ and $s$. For the sake of 
simplicity, the two lowest eigenvalues are both 0; they could as well be different? provided they are well below $E^{\lambda}$. The link ground state in $H_{\chi \lambda}=H_{\chi} \otimes H_{\lambda}$ has thus four degenerate eigenstates (the overall state must be antisymmetrical):

$$
\begin{aligned}
0 & \leftrightarrow \frac{1}{\sqrt{2}}|0\rangle_{1}|0\rangle_{2}\left(|r\rangle_{1}|s\rangle_{2}-|s\rangle_{1}|r\rangle_{2}\right) \\
0 & \leftrightarrow \frac{1}{\sqrt{2}}|1\rangle_{1}|1\rangle_{2}\left(|r\rangle_{1}|s\rangle_{2}-|s\rangle_{1}|r\rangle_{2}\right) \\
0 & \leftrightarrow \frac{1}{2}\left(|0\rangle_{1}|1\rangle_{2}+|1\rangle_{1}|0\rangle_{2}\right)\left(|r\rangle_{1}|s\rangle_{2}-|s\rangle_{1}|r\rangle_{2}\right) \\
0 & \leftrightarrow \frac{1}{2}\left(|0\rangle_{1}|1\rangle_{2}-|1\rangle_{1}|0\rangle_{2}\right)\left(|r\rangle_{1}|s\rangle_{2}+|s\rangle_{1}|r\rangle_{2}\right)
\end{aligned}
$$

To avoid triplet states of parallel spins, we introduce the Hamiltonian $H_{12}^{\chi}$ operating on the Hilbert space

$$
H_{\chi}=\operatorname{span}\left\{|0\rangle_{1}|0\rangle_{2},|1\rangle_{1}|1\rangle_{2}, \frac{1}{\sqrt{2}}\left(|0\rangle_{1}|1\rangle_{2}+|1\rangle_{1}|0\rangle_{2}\right), \frac{1}{\sqrt{2}}\left(|0\rangle_{1}|1\rangle_{2}-|1\rangle_{1}|0\rangle_{2}\right)\right\}
$$

where $|0\rangle_{1}|0\rangle_{2}$ reads: the $\sigma_{z}$ component of particle 1 is down, the same for particle 2 , etc.

$$
H_{12}^{\chi}=\left(\begin{array}{cccc}
E^{\chi} & 0 & 0 & 0 \\
0 & E^{\chi} & 0 & 0 \\
0 & 0 & 0 & 0 \\
0 & 0 & 0 & 0
\end{array}\right) \text {, with } E^{\chi} \text { discretely above } 0
$$

$H_{12}^{\chi}$ commutes with $H_{12}^{\lambda}$ and lifts the energy level of the triplet states of parallel spins. The link ground state remains two-fold degenerate and hosts the spin singlet state $|\psi\rangle_{\text {_ }}$ and the antiparallel spin triplet state $|\psi\rangle_{+}$:

$$
|\psi\rangle_{\mp}=\frac{1}{2}\left(|0\rangle_{1}|1\rangle_{2} \mp|1\rangle_{1}|0\rangle_{2}\right)\left(|r\rangle_{1}|s\rangle_{2} \pm|s\rangle_{1}|r\rangle_{2}\right)
$$

upper (lower) signs go together. With simple manipulations, (2) can be written:

$$
|\psi\rangle_{\mp}=\frac{1}{\sqrt{2}}\left(\left|\psi^{\prime}\right\rangle \mp\left|\psi^{\prime \prime}\right\rangle\right)
$$

where

\footnotetext{
${ }^{3}$ which would be necessary if we regarded $H_{12}^{\lambda}$ as acting on spatial variables.
} 


$$
\begin{gathered}
\left|\psi^{\prime}\right\rangle=\frac{1}{\sqrt{2}}\left(|0\rangle_{1}|r\rangle_{1}|1\rangle_{2}|s\rangle_{2}-|1\rangle_{1}|s\rangle_{1}|0\rangle_{2}|r\rangle_{2}\right)=|0\rangle_{r}|1\rangle_{s}, \\
\left|\psi^{\prime \prime}\right\rangle=\frac{1}{\sqrt{2}}\left(|1\rangle_{1}|r\rangle_{1}|0\rangle_{2}|s\rangle_{2}-|0\rangle_{1}|s\rangle_{1}|1\rangle_{2}|r\rangle_{2}\right)=e^{i \delta}|1\rangle_{r}|0\rangle_{s} .
\end{gathered}
$$

It is readily checked that both terms appearing in $\left|\psi^{\prime}\right\rangle\left(\left|\psi^{\prime \prime}\right\rangle\right)$ in the particle representation map onto $|0\rangle_{r}|1\rangle_{s}\left(|1\rangle_{r}|0\rangle_{s}\right)$ in qubit representation; $\delta$ is an undefined phase. Thus

$$
|\psi\rangle_{\mp}=\frac{1}{\sqrt{2}}\left(|0\rangle_{r}|1\rangle_{s} \mp|1\rangle_{r}|0\rangle_{s}\right),
$$

where we have set $\delta=0$ for $|\psi\rangle$ to behave for a rotation of the $\chi$ reference in eq. (6) as in eq. (2).

Since $|\psi\rangle_{-}$and $|\psi\rangle_{+}$are degenerate, we can say as well that the two orthogonal ground eigenstates of link $r, s$ are $|0\rangle_{r}|1\rangle_{s}$ and $|1\rangle_{r}|0\rangle_{s}$. Therefore the degenerate link ground state has the form:

$$
|\xi\rangle=\alpha|0\rangle_{r}|1\rangle_{s}+\beta|1\rangle_{r}|0\rangle_{s}
$$

with $|\alpha|^{2}+|\beta|^{2}=1$.

Let $A_{12}=1-P_{12}$ followed by renormalization be the antisymmetric operator in particle representation. By definition, the application of $A_{12}$ to a generic state filters out and normalizes its antisymmetrical component. We can see that $A_{12}\left|\psi^{\prime}\right\rangle=\left|\psi^{\prime}\right\rangle$ and $A_{12}\left|\psi^{\prime \prime}\right\rangle=\left|\psi^{\prime \prime}\right\rangle$. In equivalent terms: $A_{12}|0\rangle_{r}|1\rangle_{s}=|0\rangle_{r}|1\rangle_{s}$ and $A_{12}|1\rangle_{r}|0\rangle_{s}=|1\rangle_{r}|0\rangle_{s}$. Also: $A_{12}|0\rangle_{r}|0\rangle_{s}=|0\rangle_{r}|0\rangle_{s}$ and $A_{12}|1\rangle_{r}|1\rangle_{s}=|1\rangle_{r}|1\rangle_{s}$, if we are dealing with the excited states of energy eigenvalue $E^{\chi}$. However, such states are forbidden in the link ground state. They should not be confused with the "bad" tensor products:

$$
\begin{aligned}
& |0\rangle_{r}|0\rangle_{s}=\frac{1}{\sqrt{2}}|0\rangle_{1}|0\rangle_{2}\left(|r\rangle_{1}|s\rangle_{2}+|s\rangle_{1}|r\rangle_{2}\right), \\
& |1\rangle_{r}|1\rangle_{s}=\frac{1}{\sqrt{2}}|1\rangle_{1}|1\rangle_{2}\left(|r\rangle_{1}|s\rangle_{2}+|s\rangle_{1}|r\rangle_{2}\right),
\end{aligned}
$$

which are symmetrical and violate $A_{12}$. We should note that qubit notation, and qubit density matrices $\left(|0\rangle_{r}\left\langle\left. 0\right|_{r}\right.\right.$, etc.), on which the relaxation process operates, are the same for both kinds of states. 
All this becomes, in $H_{r s}=\operatorname{span}\left\{|0\rangle_{r}|0\rangle_{s},|0\rangle_{r}|1\rangle_{s},|1\rangle_{r}|0\rangle_{s},|1\rangle_{r}|1\rangle_{s}\right\}$, the projector (or "symmetry") $A_{r s}$ defined by the following equations:

$$
A_{r s}|\chi\rangle_{r}|1-\chi\rangle_{s}=|\chi\rangle_{r}|1-\chi\rangle_{s}, \quad A_{r s}|\chi\rangle_{r}|\chi\rangle_{s}=0
$$

with $\chi=0,1 . A_{r s}$ symmetrization projects a generic state of $H_{r s}$ onto the symmetrical subspace $H_{A_{r s}}$ spanned by the legitimate link states, given by eq. (7). It is understood that the application of $A_{r s}$ is followed by renormalization. Let $\langle L\rangle$ be the expected value of the link energy. Clearly, $A_{r s}$ projection is equivalent to the two mathematically simultaneous conditions: $A_{12}$ projection and $\langle L\rangle=0$.

\section{A divergence between one and two-way propagation models}

The link state, in the reference where $H_{12}^{\chi}$ is diagonal, is given by eq. (7). Let us consider continuous transformations of state (7) which leave $A_{r s}$ symmetry unaltered. Without significant restriction, state $(7)$ is rewritten:

$$
|\xi\rangle=\cos \vartheta|0\rangle_{r}|1\rangle_{s}+\sin \vartheta|1\rangle_{r}|0\rangle_{s}
$$

There is of course a unitary operator $R(\varphi)$, acting on the Hilbert space $H_{r s}=$ $\operatorname{span}\left\{|0\rangle_{r}|0\rangle_{s},|0\rangle_{r}|1\rangle_{s},|1\rangle_{r}|0\rangle_{s},|1\rangle_{r}|1\rangle_{s}\right\}$, which changes $|\xi\rangle$ of eq. (9) into:

$$
\left|\xi^{\prime}\right\rangle=\cos (\vartheta+\varphi)|0\rangle_{r}|1\rangle_{s}+\sin (\vartheta+\varphi)|1\rangle_{r}|0\rangle_{s}
$$

without ever (for all $\varphi$ ) violating $A_{r s}$ symmetry; here $\varphi=\omega t$. Since $\vartheta$ and $\varphi$ will be used to model the result of gate relaxation, we can assume $\vartheta, \varphi \geqslant 0$ and $0 \leqslant \vartheta+\varphi \leqslant \frac{\pi}{2}$.

It could be seen that $R(\varphi)$ operates on the overall link state in an irreducible way. However, for reasons that will become clear, we are interested in a factorizable operator: $R_{r s}(\varphi)=R_{r}(\varphi) R_{s}(\varphi)$, where $R_{r}(\varphi)\left[R_{s}(\varphi)\right]$ operates on the state of qubit $r(s)$, independently of the state of qubit $s(r)$. Let $R_{t}(\varphi) \equiv\left(\begin{array}{cc}\cos \varphi & -\sin \varphi \\ \sin \varphi & \cos \varphi\end{array}\right)$, with $t=r, s$.

We should note that $R_{r s}(\varphi)$ does not commute with $A_{r s}$. Its application to state $(9)$ introduces the terms $|0\rangle_{r}|0\rangle_{s}$ and $|1\rangle_{r}|1\rangle_{s}$. There are two possible cases: 
(i) these terms are the excited states which satisfy $A_{12}$, then the link goes into a superposition of two energy eigenstates, ground and $E^{\chi}$, while its expected energy $\langle L\rangle$ is raised, and

(ii) these terms are the symmetrical states of parallel spin, which violate $A_{12}$; such states would be, so to speak, immediately projected off.

In this latter case $R_{r s}(\varphi)$ operates adiabatically on the link, leaving it in its ground eigenstate, where $A_{r s}$ is satisfied Further below, it will become clear that this is a possible solution.

We shall formalize this, first in the conventional one-way propagation model. A series of infinitesimal operations $R_{r s}(\delta \varphi)$, each followed by $A_{r s}$ symmetrization should be applied until $\int \delta \varphi=\varphi$. However this operation suffers from a Zeno effect which keeps state (9) unaltered: indeed $\lim _{n \rightarrow \infty}\left[A_{r s} R_{r s}\left(\frac{\varphi}{n}\right)\right]^{n}|\xi\rangle=|\xi\rangle$, as it can be checked. In fact, in first order, $R_{r s}(\varphi)$ drives the link state out of the symmetrical subspace $H_{A_{r s}}=\operatorname{span}\left\{|0\rangle_{r}|1\rangle_{s},|1\rangle_{r}|0\rangle_{s}\right\}$ orthogonally to it. $A_{r s}$ projection on $H_{A_{r s}}$ brings the link state back to the starting point. Therefore $R_{r s}(\varphi)$ cannot operate adiabatically in a one-way propagation perspective, or in calculus which is naturally one-way. It would inevitably lift $\langle L\rangle$.

However, calculus may not be applicable to a situation which involves a continuous projection due to $A_{r s}$ symmetrization. As a matter of fact, during an elementary rotation $\triangle \varphi=\frac{\varphi}{n}$, symmetry $A_{r s}$ is continuously violated (if the link remains in its ground state, the fundamental symmetry $A_{12}$ is violated). This error may not vanish in the limit $\triangle \varphi \rightarrow 0$.

A reasonable alternative is to think that rotation and symmetrization should be applied in a mathematically simultaneous way, so that $A_{r s}$ is never violated. This removes the Zeno effect and brings in a two-way propagation (Section II.F). We consider a free normalized vector $\left|\xi^{\prime \prime}\right\rangle$ of the Hilbert space $H_{r s}$. By this we mean that its amplitudes on $H_{r s}$ basis vectors are free variables (up to normalization) independent of each other. This vector

\footnotetext{
${ }^{4}$ For a study of adiabatic operation on energy eigenstates, see [33].
} 
must satisfy, for all $\varphi$ or $t(\varphi=\omega t)$, the following system of mathematically simultaneous equations:

(i) $A_{r s}\left|\xi^{\prime \prime}\right\rangle=\left|\xi^{\prime \prime}\right\rangle$,

(ii) $\operatorname{Tr}_{s}\left|\xi^{\prime \prime}\right\rangle\left\langle\xi^{\prime \prime}\left|=\cos ^{2}(\vartheta+\varphi)\right| 0\right\rangle_{r}\left\langle\left. 0\right|_{r}+\sin ^{2}(\vartheta+\varphi) \mid 1\right\rangle_{r}\left\langle\left. 1\right|_{r}\right.$,

(iii) $\operatorname{Tr}_{r}\left|\xi^{\prime \prime}\right\rangle\left\langle\xi^{\prime \prime}\left|=\sin ^{2}(\vartheta+\varphi)\right| 0\right\rangle_{s}\left\langle\left. 0\right|_{s}+\cos ^{2}(\vartheta+\varphi) \mid 1\right\rangle_{s}\left\langle\left. 1\right|_{s}\right.$.

Condition (i) ensures that $A_{r s}$ is never violated and is equivalent to $A_{12}\left|\xi^{\prime \prime}\right\rangle=\left|\xi^{\prime \prime}\right\rangle$ and $\langle L\rangle=0$. Conditions (ii) and (iii) mean that the density matrix of each qubit has been rotated by $\varphi$. For all $\varphi$, the above system of mathematically simulteneous equations yields the solution:

$$
\left|\xi^{\prime \prime}\right\rangle=\cos (\vartheta+\varphi)|0\rangle_{r}|1\rangle_{s}+e^{i \delta} \sin (\vartheta+\varphi)|1\rangle_{r}|0\rangle_{s}
$$

as it is readily checked. The further condition that $\left\|\left\langle\xi \mid \xi^{\prime \prime}\right\rangle\right\|$ be maximum (see Section II.F), sets $\delta=0$, thus yielding to state (10).

As we will see (Section II.F), this is exactly an application of a two-way propagation model fo the projection process induced by $A_{r s}$ (by $A_{12}$ under an adiabatic operation). To sum up, in this scheme, one can operate separately and adiabatically on the link qubits; the link state undergoes a unitary evolution while remaining in its degenerate ground state, thus always satisfying $A_{r s}$. This unitary evolution is driven by conditions (ii), (iii) and forged by condition (i), namely continuous $A_{r s}$ projection. The fact that the resulting evolution is unitary thus reversible (it does not dissipate free energy), is consistent with the assumption of adiabatic operation.

We will show another way of obtaining the same result. Let qubit $r$ be connected to the one-qubit Hamiltonian $\left(\begin{array}{cc}E_{r} & 0 \\ 0 & 0\end{array}\right)_{r}$ operating on span $\left\{|0\rangle_{r},|1\rangle_{r}\right\}$ and qubit $s$ be connected

\footnotetext{
${ }^{5}$ We will use the model developed in $[18,19]$, whose outline will be given in Section II.F.
} 
to the one-qubit Hamiltonian $\left(\begin{array}{cc}0 & 0 \\ 0 & E_{s}\end{array}\right)_{s}$ operating on span $\left\{|0\rangle_{s},|1\rangle_{s}\right\}$. Disregarding $A_{r s}$, the two qubits would relax independently of each other onto $|1\rangle_{r}|0\rangle_{s}$ (a heat bath should provide for both "shaking" and "dumping").

The expected energy of the two gates in state (9) (the initial condition) is $E_{1}=$ $\left(E_{r}+E_{s}\right) \cos ^{2} \vartheta$. Now we set the final condition that the state of this network simultaneously satisfies $A_{r s}$ and has a lower expected energy, say $E_{2}=\left(E_{r}+E_{s}\right) \cos ^{2}(\vartheta+\varphi)$. We assume that the energy difference has gone to the heat bath. This yields to state (10) again, as it can be checked. Now the unitary evolution from (9) to (10) is driven by the independent gate relaxation process, and of course it is forged by condition (i).

One can think that gate relaxation may not always operate adiabatically on the link. The excited (antisymmetrical) components $|0\rangle_{r}|0\rangle_{s}$ and $|1\rangle_{r}|1\rangle_{s}$ would appear in a superposition with the link ground state. However, assuming $E_{r}, E_{s} \ll E^{\chi}$, these components would have a small amplitude and should consequently be short lived, due to the continuous gate relaxation. The above two-way evolution would still apply to the Everett worlds where the link is in its symmetric ground eigenstate.

We should further note that, rotating either independent qubit is equivalent to rotating both; the two rotations are redundant with respect to one another. This can be checked by removing either condition (ii) or (iii); just one is sufficient to yield state (10).

We shall now go back to the one-way model. It can be checked that there is no way of operating separately and adiabatically on the two qubits to perform a unitary transformation of the link state inside the Hilbert space $H_{A_{r s}}$. Gate relaxation will raise the link expected energy $\langle L\rangle$ by some amount, non-infinitesimal this time. Therefore the link state can undergo a rotation. Then the link would relax on ground restoring $A_{r s}$ on the rotated state. This would go on repeatedly. However, this form of quantum annealing works exactly like classical annealing. 


\section{E. Turning Gate Dynamics on}

To sum up, having turned link Hamiltonians on and allowed links to relax onto ground (independently of each other), the entire lattice of qubits $L$ is obtained, where qubits eigenvalues are submitted to the symmetry (affecting any pair of qubits connected by a link) $\tilde{A}=\prod_{r, s} A_{r s}$. Note that all $A_{r s}$ operate on disjoint Hilbert spaces, concerning the states of disjoint pairs of nodes, and are pairwise commuting.

After creating $L$ and $\tilde{A}$, gate Hamiltonians are "turned on", then gates are allowed to relax. Here we consider the network at equilibrium, when all gates and links are in ground state. How this state is reached will be the subject of Section II.F.

Let $P_{g}$ be the projector on the ground state of gate $\# g$, and $|\Psi\rangle=\sum_{h} \alpha_{h}\left|\Psi_{h}\right\rangle$ the generic network state, where $\left|\Psi_{h}\right\rangle$ is a tensor product of all qubit eigenstates. At equilibrium, when all gates are in ground state, for all $P_{g}$ each $\left|\Psi_{h}\right\rangle$ should satisfy $P_{g}\left|\Psi_{h}\right\rangle=\left|\Psi_{h}\right\rangle$. This yields to $\left|\Psi_{h}\right\rangle$ s which satisfy all gates. Each $\left|\Psi_{h}\right\rangle$ should also satisfy $\tilde{A}\left|\Psi_{h}\right\rangle=\left|\Psi_{h}\right\rangle$. This yields to $\left|\Psi_{h}\right\rangle$ s which satisfy all links. All $P_{g}$ operate on disjoint sets of qubits, thus on disjoint Hilbert spaces, and are pairwise commuting. Let $P=\Pi_{g} P_{g}$, where $g$ runs over all gate labels. The foregoing simultaneous equations are summarized by:

$$
\tilde{A} P|\Psi\rangle=|\Psi\rangle
$$

Any $\left|\Psi_{h}\right\rangle$ satisfying eq. (11) satisfies all gates and links and is thus a solution (for the case with no solution, see Section II.A). Substituting $\tilde{A} P$ with $P \tilde{A}$ yields to the same conclusion. Even if $\tilde{A}$ and $P$ did not commute, the network ground states are eigenstates of both.

However, the propagator of the actual relaxation process is shaped by $\tilde{A}$ and commutes with it.

This approach would suffer no interaction with the environment provided $L$ and $\tilde{A}$ remain in place: entanglement with a reservoir initially in state $|R\rangle$ changes each $\left|\Psi_{h}\right\rangle|R\rangle$ into $\left|\Psi_{h}\right\rangle\left|R_{h}\right\rangle$, where $\left|R_{h}\right\rangle$ is now correlated with $\left|\Psi_{h}\right\rangle$, without altering the selection performed by eq. (11). 


\section{F. Relaxation Time}

We shall now discuss relaxation time. Let us consider a simple "network" of just one link $r, s$ and one qubit gate, whose Hamiltonian operates on span $\left\{|0\rangle_{s},|1\rangle_{s}\right\}$ and has the form $\left(\begin{array}{cc}E_{s} & 0 \\ 0 & 0\end{array}\right)_{s}$. Let the initial state of this network be the symmetrical state $\left|\Psi\left(t_{1}\right)\right\rangle=|0\rangle_{r}|1\rangle_{s}$. For the sake of simplicity, only qubit $r$ is assumed to be in direct interaction with a heat bath. At equilibrium, the (symmetrical) ground state should instead be $|1\rangle_{r}|0\rangle_{s}$.

We shall apply the one-way propagation model first. We will limit ourselves to examining the network initial evolution from $|0\rangle_{r}|1\rangle_{s}$. This is readily done and provides useful insight. Since the initial state is factorized, relaxation of qubit $s$ and heat bath perturbation on qubit $r$ are (initially and in first approximation) independent of each other.

The initial independent relaxation of gate s can be modeled by the two-step evolution:

$$
\left|\Psi\left(t_{1}\right)\right\rangle_{s}=|1\rangle_{s} \rightarrow\left|\Psi\left(t_{2}\right)\right\rangle_{s}=\cos \triangle \varphi|1\rangle_{s}+e^{i \delta} \sin \triangle \varphi|0\rangle_{s}
$$

where $t_{2}>t_{1}$, and $\delta$ is an undefined phase due to entanglement with the outside world. We are using the method of random phases: the average over $\delta$ of the density matrix corresponding to the state on the right of the arrow, yields $\cos ^{2} \triangle \varphi|1\rangle_{s}\left\langle\left. 1\right|_{s}+\sin ^{2} \triangle \varphi \mid 0\right\rangle_{s}\left\langle\left. 0\right|_{s}\right.$, as expected.

The initial independent heat bath perturbation on qubit $r$ can be modeled by the twostep evolution:

$$
\left|\psi\left(t_{1}\right)\right\rangle_{r}=|0\rangle_{r} \rightarrow\left|\psi\left(t_{2}\right)\right\rangle_{r}=\cos \triangle \vartheta|0\rangle_{r}+e^{i \delta^{\prime}} \sin \triangle \vartheta|1\rangle_{r}
$$

where $\triangle \vartheta$ is a random phase.

We can approximate the network initial evolution by symmetrizing the tensor product of the two qubits:

$$
\begin{aligned}
\left|\psi\left(t_{1}\right)\right\rangle & =|0\rangle_{r}|1\rangle_{s} \rightarrow \\
\left|\psi\left(t_{2}\right)\right\rangle & =\cos \triangle \varphi \cos \triangle \vartheta|0\rangle_{r}|1\rangle_{s}+e^{i \delta^{\prime \prime}} \sin \triangle \varphi \sin \triangle \vartheta|1\rangle_{r}|0\rangle_{s}
\end{aligned}
$$


up to normalization; here $\delta^{\prime \prime}=\delta+\delta^{\prime}$.

This is an interesting result. If $\triangle \vartheta=0$, the evolution remains frozen in its initial state $|0\rangle_{r}|1\rangle_{s}$ (see eq. 14). The relaxation of gate $s$ is conditioned by the action of the heat bath on qubit $r$. This means that, so to speak, by a lucky coincidence the network can relax, otherwise it cannot. Likely, this would mean frustration in a more complex network. In this approximation, one can see no reason why quantum annealing should perform better than classical annealing.

Before applying the two-way propagation model $(T W)$ developed in $[18,19]$, we shall outline it. This model was meant to justify the projection undergone by a quantum state during various measurement operations.

Both forward and backward propagations are referred to the same direction of time in the episystem, on the basis of the following argument. Time can be seen as a parameter in the endosystem. It is the direction of causality in the endosystem - either forward or backward in time - that makes the difference between the two propagations. Therefore, both propagations are represented by kets evolving forward in time: one associated with causality forward in time, the other with causality backward in time. Because of the absence of a privileged direction of causality, the two propagations should be indistinguishable.

Indistinguishability requires that (i) each propagation undergoes the same transformations of the conventional one-way propagation, up to an overall phase, which is irrelevant to each propagation, and (ii) the relative phase of the two propagations is either 0 or $\pi$.

As a consequence of indistinguishability, the two propagations should proceed in coherent superposition. This superposition is the complete description of the propagation. Both initial and final conditions, comprising normalization, should be applied to it, whereby the initial (or indifferently) final condition of each propagation is just a free un-normalized state (completely undefined before the application of all conditions) of the system Hilbert space.

State projection (due to either a measurement or a watchdog effect) implies a $\pi$ shift in the relative phase of the two propagations. Consequently, their superposition changes 
from constructive to destructive interference or vice-versa. Clearly, this can justify the discontinuous evolution of the quantum state throughout a projection.

The foregoing model yields to the following simple rules to be applied to the superposition (the complete description):

(i) the state before reduction $\left|\psi\left(t_{1}\right)\right\rangle$ coincides with the usual state;

(ii) the state after reduction $\left|\psi\left(t_{2}\right)\right\rangle$ is a free normalized state of the system Hilbert space, subject to the final conditions and to the supplementary condition that

(iii) $\left\|\left\langle\psi\left(t_{1}\right) \mid \psi\left(t_{2}\right)\right\rangle\right\|$ is maximum.

A preparation, or a propagation thereof, can be the state before projection. The final conditions can be set from the future, for example they can be the result of partial or complete measurement, unforeseeable from the past.

As it should be clear now, in Section II.D we have applied the two-way propagation model schematized above. The result was that $A_{r s}$ works like interference (destructive due to projection, constructive due to renormalization) on the relaxation of independent gates.

We shall now apply the $T W$ model to the simple network introduced at the beginning of this Section.

The independent relaxation of gate $s$ is modeled by the two-step evolution (12). The gate energy in $\left|\psi\left(t_{2}\right)\right\rangle_{s}$ is $E_{s} \cos ^{2} \Delta \varphi$ (from now on, by energy in a state we will mean the expected value of the energy operator in that state).

The heat bath perturbation on qubit $r$ is modeled by (13). Let the operator $\left(\begin{array}{cc}E_{r} & 0 \\ 0 & -E_{r}\end{array}\right)_{r}$ represent the energy of the qubit-heat bath interaction. Its expected value in $\left|\psi\left(t_{2}\right)\right\rangle_{r}$ is $E_{r} \cos 2 \triangle \vartheta . \quad \Delta \vartheta$ can be assumed to be a random variable with uniform distribution in $[0,2 \pi]$, consequently $\cos 2 \Delta \vartheta$ is zero on the average. We further assume $E_{r} \ll E_{s}$.

Since the gate and the heat bath operate independently (given that $A_{r s}$ works as interference), the network energy at time $t_{2}$ is $E=E_{s} \cos ^{2} \triangle \varphi+E_{r} \cos 2 \triangle \vartheta$. 
The network state at time $t_{2}$ is a free normalized state of the network Hilbert space, submitted to the final conditions:

(i) $\left|\Psi\left(t_{2}\right)\right\rangle$ satisfies $A_{r s}$;

(ii) the energy in $\left|\psi\left(t_{2}\right)\right\rangle$ is $E$.

This yields the two-step evolution

$$
\left|\psi\left(t_{1}\right)\right\rangle=|0\rangle_{r}|1\rangle_{s} \rightarrow\left|\psi\left(t_{2}\right)\right\rangle=\cos \Delta \varphi^{\prime}|0\rangle_{r}|1\rangle_{s}+e^{i \delta^{\prime \prime}} \sin \Delta \varphi^{\prime}|1\rangle_{r}|0\rangle_{s},
$$

where $\triangle \varphi^{\prime}$ is still to be determined. The former condition (iii) is irrelevant here.

The energy in $\left|\psi\left(t_{2}\right)\right\rangle$ is $E_{s} \cos ^{2} \triangle \varphi^{\prime}+E_{r} \cos 2 \triangle \varphi^{\prime}$. Therefore $\triangle \varphi^{\prime}$ must be a solution of the equation

$$
E_{s} \cos ^{2} \triangle \varphi^{\prime}+E_{r} \cos 2 \triangle \varphi^{\prime}=E_{s} \cos ^{2} \triangle \varphi+E_{r} \cos 2 \triangle \vartheta
$$

It can be readily checked that there is a unique solution in the interval of interest. If $E_{r} \ll E_{s}, \triangle \varphi^{\prime}$ - i.e. network relaxation - closely follows the independent gate relaxation $\triangle \varphi$. Moreover, $\triangle \varphi=\triangle \varphi^{\prime}$ on the average.

This is quite different from the one-way model. Since the network energy is the sum of the energies of the independent network elements, frustration is never met. For example, the absence of a heat bath $\left(\triangle \vartheta\right.$ or $\left.E_{r}=0\right)$ does not freeze relaxation, unlike in the one-way model.

This can be generalized to any Boolean network. Condition (iii), moving in time together with continuous projection, allocates the overall energy among the network degrees of freedom. Still, the overall energy decreases as if all network elements relaxed independently of each other and frustration is never met. Although a dynamic model is still lacking, this qualitative difference should yield an essential advantage over classical simulated annealing.

Let us note that evolution (15) can represent an identical rotation of the two independent qubits $r$ and $s$, obtained by rotating just qubit $s$. We must put $E_{r}=0$ (thus obtaining $\triangle \varphi^{\prime}=\triangle \varphi$ ) and $\delta^{\prime \prime}=0$, which ensures rotation additivity; this is legitimate since we are now dealing with a pure state rotation. We find again the result of Section II.D. 


\section{DISCUSSION}

We have developed a form of quantum annealing computation where network wiring is implemented by a quantum symmetry $\tilde{A}$ related to particle statistics. How this quantum wiring operates can be interpreted in two different ways.

In a one-way, conventional, propagation model, this wiring does not exhibit any special feature with respect to classical wiring. Disregarding tunnelling, this quantum model does not yield any improvement over classical annealing.

In an unconventional - two-way - propagation model, particle statistics works as a projection. $\tilde{A}$ would continuously project the state of the independently relaxing gates on a "symmetric" subspace where network wiring is satisfied. This is naturally a blunt conjecture - this work should be considered an exploration of the possible role played by particle statistics (viewed as projection) in quantum computation.

We should remark that something similar appears in other situations involving particle statistics. For example, given two non-interacting identical spin $\frac{1}{2}$ fermions whose spatial wave functions overlap, there is a correlation between their mutual distance and the character of their spin state, either singlet or triplet. This correlation, due to antisymmetrization, creates a sort of "wiring" between two otherwise independent parts.

Although an implementation model is still lacking, we see no reason to think that this is out of reach. In the example where $\lambda$ labels the sites of a spatial lattice and $\chi$ labels the

$\sigma_{z}$ component of the spin $\frac{1}{2}$ particle hosted by each site, the link behaviour is likely to be related to exchange interaction.

Were this model implementable - for example in the case of the single link and a one qubit Hamiltonian (Section II.F) - relaxation speed could be tested. In principle, this should decide which propagation model - either one or two-way - applies.

The hypothesis of a computation speed-up based on chronology violation links this work with D. Deutsch's paper on computation along closed time-like lines. From that paper we quote: "it is curious that the analysis of a physical situation which might well not 
occur should yield so many insights in quantum theory. But that is the nature of thought experiments. Perhaps we should also bear in mind that a frequently observed effect of time is to convert thought experiments into real ones". Placing chronology violation within the time-energy uncertainty relation and the projection of an evolving state induced by particle statistics, might be a step towards feasibility.

This research has been supported by Elsag Bailey, a Finmeccanica company. Thanks are due to A. Ekert, D. Finkelstein, L. Levitin, S. Lloyd, D. Monti, M. Rasetti and T. Toffoli for the useful suggestions.

[1] C.H. Bennett, "Logical Reversibility of Computation" IBM J. Res. Dev. 6, 525 (1979).

[2] E. Fredkin and T. Toffoli, Int. J. Theor. Phys. 21, 219 (1982).

[3] C.H. Bennett, Int. J. Theor. Phys. 21, 905 (1982).

[4] T. Toffoli, Math. Syst. Theory 14, 13 (1981).

[5] P. Benioff, Phys. Rev. Letters 48, 1581 (1982).

[6] R.P. Feynman, Opt. News 11, 11 (1985).

[7] D. Deutsch, Proc. Roy. Soc. London A 400, 97, (1985).

[8] W.H. Zurek, Phys. Rev. Letters 4, 391 (1984).

[9] A. Peres, Phys. Rev. A 32, 3266 (1985).

[10] D. Deutsch and R. Jozsa, Proc. Roy. Soc. London A 439, 553 (1992).

[11] E. Bernstein and U. Vazirany, "Quantum Complexity Theory", Proceedings of the 25th Annual ACM Symposium on Theory of Computing 11 (1193).

[12] A. Berthiaume and G. Brassard, "Oracle Quantum Computing", Proceedings of the Workshop 
on Physics and Computation - Phys. Comp. '92, IEEE Press 195 (October 1992).

[13] D.R. Simon, Proceedings of the 35th Annual Symposium on the Foundation of Computer Science, Los Alamitos, CA, 116 (1994).

[14] P.W. Shor, Proceedings of the 35th Annual Symposium on the Foundation of Computer Science, Los Alamitos, CA, 124 (1994).

[15] Y. Aharanov, P.G. Bergmann, J.L. Lebowitz, Phys. Rev. B 134, 1410 (1964).

[16] Y. Aharanov and L. Vaidman, Phys. Rev. A 41, 11 (1990).

[17] J.G. Cramer, Rev. Mod. Phys., 58, 647 (1986).

[18] G. Castagnoli, Int. J. Theor. Phys., 34, 1283 (1995).

[19] G. Castagnoli and M. Rasetti, Int. J. Mod. Phys. B 10, 296 (1996).

[20] C. Bennett et al. "Strengths and Weaknesses of Quantum Computation", unpublished material (1994).

[21] A. Berthiaume, D. Deutsch and R. Jozsa, Proceedings of the Workshop on Physics and Computation, Phys. Comp. 94 (IEEE Computer Society Press, Los Alamitos, CA 1994)

[22] A. Ekert, C. Macchiavello, Phys. Rev. Letters, 77, 2585 (1996).

[23] P. Shor, Phys. Rev. A, 52, 2493 (1995).

[24] P. Shor, D. Di Vincenzo, Phys. Rev. Letters, 77, 3260 (1966).

[25] D.R. Finkelstein, "Quantum Relativity", Springer Verlag Berling Herdelberg, 1996.

[26] D. Deutsch, Phys. Rev. D 44, 3197 (1991).

[27] S. Kirkpatrick and B. Selman, Science 264, 1297 (1994), Artificial Intelligence 81, 106 (1996).

[28] G. Castagnoli and M. Rasetti, Int. J. Theor. Phys., 32, 2335 (1993).

[29] A. Barenco, D. Deutsch, A. Ekert, R. Jozsa, Phys. Rev. Lett. 74, 4083 (1995). 
[30] D.P. Di Vincenzo, Phys. Rev. A 50, 1015 (1995).

[31] A. Barenco, D. Deutsch, A. Ekert, Proc. R. Soc. London A 449, 669 (1995).

[32] S. LLoyd, Phys.Rev.Lett. 75, 346 (1995).

[33] Y. Aharanov and L. Vaidman, Ann. NY Acad. Sci. 480, 361 (1995). 


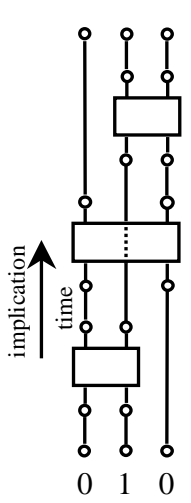

(a)

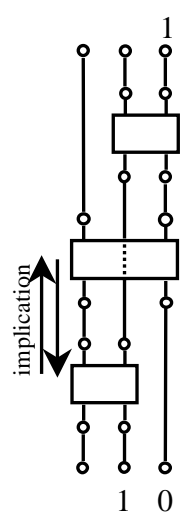

(c)

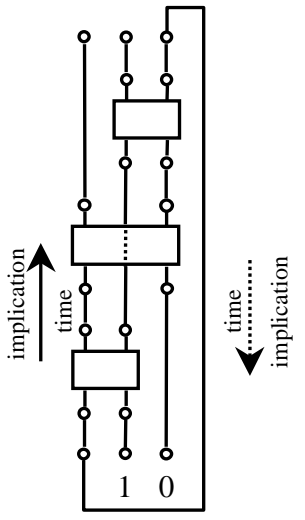

(b)

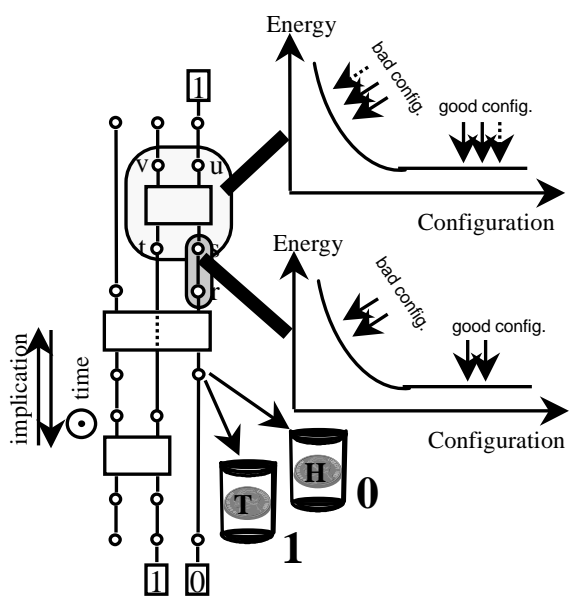

(d)

Fig. 1 